\title{
Spectrum of MRI abnormalities in pediatric seizures
}

\section{Mathur M. ${ }^{1}$, Jeet Singh S. ${ }^{2 *}$, Kaur N. ${ }^{3}$, Mittal D. ${ }^{4}$, Garg Y. ${ }^{5}$, Raikhy A. \\ DOI: https://doi.org/10.17511/ijmrr.2020.i01.11}

\footnotetext{
1 Manoj Mathur, Professor, Department of Radiodiagnosis, Govt. Medical College and Rajindra Hospital, Patiala, Punjab, India.

2* Simran Jeet Singh, Junior Resident, Department of Radiodiagnosis, Govt. Medical College and Rajindra Hospital, Patiala, Punjab, India.

3 Navpreet Kaur, Senior Resident, Department of Pediatrics, Post Graduate Institute of Medical Education \& Research, Chandigarh, Punjab, India.

4 Dimple Mittal, Junior Resident, Department of Radiodiagnosis, Govt. Medical College and Rajindra Hospital, Patiala, Punjab, India.

5 Yogesh Garg, Junior Resident, Department of Radiodiagnosis, Govt. Medical College and Rajindra Hospital, Patiala, Punjab, India.

6 Ankit Raikhy, Consultant Minimal Access Surgery, Department of Radiodiagnosis, Govt. Medical College and Rajindra Hospital, Patiala, Punjab, India.
}

Background: Seizure is a pediatric emergency. Accurate determination of the etiology of seizures is very important to start an effective treatment. The study aims to determine the spectrum of Imaging abnormalities by Magnetic Imaging Resonance (MRI) in children who presented with seizures. Methods: It is a hospital-based prospective observational study which was carried out in Government Medical College and Rajindra Hospital, Patiala. This study included 50 pediatric patients in the age group between 0 months to 18 years who were referred to the Department of Radiodiagnosis for brain MRI between October 2017 to September 2019. Results: Neuroimaging abnormality was found in $19(38 \%)$ cases. $31(62 \%)$ patients had no abnormal finding. The most common imaging findings were inflammatory granuloma in $5(10 \%)$ patients. Other findings wereHypoxic-ischemic injury (HII) in $4(8 \%)$, Mesial temporal sclerosis in $2(4 \%)$, cerebral atrophy in $1(2 \%)$, Hemorrhage in $1(2 \%)$, Tuberous sclerosis in $1(2 \%)$, Focal cortical dysplasia in $1(2 \%)$, Lissencephaly in $1(2 \%)$, Joubert syndrome in $1(2 \%)$, and Arachnoid cyst in 1 (2\%) patients. Conclusion: The MRI was able to identify brain lesions in $38 \%$ of pediatric patients who presented with seizures. The study revealed inflammatory granuloma as the commonest cause of seizures in children, followed by Hypoxic-Ischemic Injury. Early recognition of potentially treatable diseases helps in timely treatment and arrest of disease progression. It is recommended to use MRI as a primary investigation during the evaluation and management of pediatric seizures.

Keywords: Hypoxic ischemic injury, Inflammatory granuloma, Neurocysticercosis, Seizures

Corresponding Author

Simran Jeet Singh, Junior Resident, Department of Radiodiagnosis, Govt. Medical College and Rajindra Hospital, Patiala, Punjab, India.

Email: drsidhu456@gmail.com
How to Cite this Article

To Browse
Mathur M, Singh SJ, Kaur N, Mittal D, Garg Y, Raikhy A. Spectrum of MRI abnormalities in pediatric seizures. Int J Med Res Rev. 2020;8(1):69-75. Available From https://ijmrr.medresearch.in/index.php/ijmrr/article/ view/1120

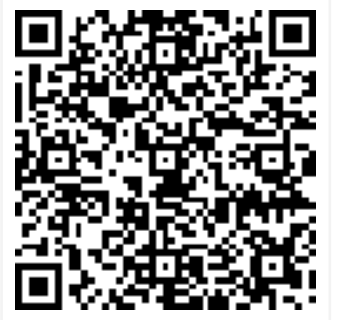

Manuscript Received 2020-01-04

Conflict of Interest No
Review Round 1 2020-01-24

Funding

$\mathrm{Nil}$
Review Round 2 2020-01-30

Ethical Approval Yes
Review Round 3

Plagiarism X-checker $4 \%$
Accepted 2020-02-04

Note

(C) 2020 by Manoj Mathur, Simran Jeet Singh, Navpreet Kaur, Dimple Mittal, Yogesh Garg, Ankit Raikhy and Published by Siddharth Health Research and Social Welfare Society. This is an Open Access article licensed under a Creative Commons Attribution 4.0 International License https://creativecommons.org/licenses/by/4.0/ unported [CC BY 4.0]. 


\section{Introduction}

A seizure is a common cause for hospitalization of children and results in significant mortality and morbidity [1]. A seizure is a clinical problem that occurs in $10 \%$ of pediatric patients $[2,3]$. During the first month of life, a seizure is the most common neurological presentation and may lead to adverse long-term outcomes [4-7]. A specific etiology for the seizures can be better detected in infants as compared to children [8]. However, in $12-15 \%$ of neonates, the etiology remains unknown [9-11]. The intent of performing a neuroimaging study in a pediatric patient is to recognize a serious condition that may need immediate intervention [3]. In some parts of the world, lack of health care facilities is a major issue. In other regions for example, in many parts of India, infrastructure does exist, but the appropriate health services are not always accessible to the people [12]. The prevalence rate of epilepsy in India has been estimated to be $9 / 1000$ of the general population [13]. Up to $40 \%$ of children with epilepsy would not attain freedom from seizures with antiepileptic drugs (AEDs). Early diagnosis of refractory seizures is quite important, as is recognition of the next appropriate therapeutic step for the patient [14]. Seizures are usually related to significant illness, sometimes requiring specific therapy. Seizures may interfere with cardiorespiratory function as well as nutrition and can have adverse long-term effects on cerebral development. Following are the potential mechanisms leading to brain injury with recurrent neonatal seizures:- 1) Hypoventilation/apnea resulting in hypoxia (leading to cardiovascular collapse), decreased cerebral blood flow [CBF] and enhanced risk of hypoxic-ischemic injury), or hypercarbia (leading to increase in CBF and increased risk of intracranial hemorrhage ( $\mathrm{ICH}) .2$ ) Elevated blood pressure increases CBF (Cerebral Blood Flow) and thus increase risk of intracranial hemorrhage. 3) Increased release of excitatory amino acids results in excitotoxic brain injury. 4) Increased glycolysis leads to hypoglycemia which thus aggravates seizure-induced brain injury. 5) Neonatal seizures vary with age of gestation. There is an increased incidence of intracranial hemorrhage in the extremely preterm neonate. In addition to it, the occurrence of seizures may also depend on the exposure to external stimuli (e.g. incubation time for some infections, initiation of feeds for some metabolic disorders, etc.). Therefore, it is crucial to modify the investigative plan according to the
Gestational age as well as the postnatal age of the infant. Most of these seizure episodes can be averted with good intensive care and control of the seizures [15-18]. Amongst the seizure patients, more than $30 \%$ of neonates who survived suffered cognitive deficits \& neurodevelopmental delay and epilepsy in later life [19-21]. Emergent management of afebrile seizure focuses on initial stabilization and searching for a cause requiring immediate intervention, such as a metabolic derangement or any other intracranial process. Not much evidence is available to favor routine imaging for pediatric patients with their first afebrile seizure having no risk factors for non -idiopathic epilepsy [22]. Further evaluation should be done, focus on history, clinical examination, and preliminary laboratory parameters to see if the event was an epileptic attack and if there is a requirement of any treatment. In those patients who are stable, MRI is the study of choice because of its sensitivity in detecting the parenchymal pathology [22]. Magnetic resonance imaging (MRI) is the main investigation to identify structural abnormalities which may be the underlying cause for seizures in neonates and it has a more sensitivity to detect subtle changes as compared to neurosonogram or CT scan [23]. MRI of the brain has become a standard imaging modality for pediatric seizures in developed countries over a period of time but not yet in developing countries due to the shortage of availability of equipment and expertise. MRI does not only help to detect the underlying brain etiology of seizure but also helps in predicting the prognosis [23]. Data on MRI findings pediatric seizures are sparse in developing countries. Hence the objective of the study is to look at the spectrum of lesions on MRI in pediatric patients presenting with seizures.

\section{Materials and Methods}

It is a prospective observational study which was carried out in Government Medical College and Rajindra Hospital, Patiala. The study population consisted of 50 pediatric patients with seizures in the age group of 0 to 18 years who were referred from clinical departments to the department of Radiodiagnosis, Rajindra hospital Patiala. All the images were obtained by $1.5 \mathrm{~T}$ MRI machine (siemens magnetom area). Both Conventional MRI and Epilepsy Protocol sequences were done. The following MRI Brain sequences were obtained for the study:- Axial T1 weighted images; axial, sagittal and coronal T2 weighted images, Axial T2 FLAIR (Fluid attenuated inversion Recovery), axial DWI 
(Diffusion-weighted images) and SWI (Susceptibility weighted imaging), images. Epilepsy protocol included- Coronal T1 Inversion Recovery images, Coronal Turbo spin-echo T2 weighted images, Axial and Coronal T1 weighted MPRAGE (Magnetized Prepared Rapid Gradient Echo).

Study Analysis: The data was described in terms of range; mean \pm standard deviation ( \pm SD), frequencies (number of cases) and relative frequencies (percentages) as appropriate. For comparing categorical data, Chi-square $(x 2)$ test was performed and the exact test was used when the expected frequency is less than 5. A probability value ( $p$-value) less than 0.05 was considered statistically significant. All statistical calculations were done using SPSS (Statistical Package for the Social Science) SPSS 21 version statistical program for Microsoft Windows.

\section{Results}

The present study consisted of 50 pediatric patients with seizures in the age group of 0 to 18 years who were referred from clinical departments to the department of Radiodiagnosis, Rajindra hospital Patiala.

Distribution of patients according to age: Out of 50 pediatric patients in the study, maximum patients (38\%) belonged to the age group 0-1 year. The second most common age group was 7-12 years. There were $19(38 \%)$ cases in the $0-1$ year age group, 12 (24\%) cases in 1-6 years age group, $13(26 \%)$ cases in 7-12 years age group and 6 $(12 \%)$ cases in more than 12 years age group.

Distribution of patients according to gestational age at birth: In the present study, out of 50 pediatric patients, $13(26.0 \%)$ were born preterm and 37 (74.0\%) were born at term.

Distribution of patients according to the history of perinatal asphyxia: Out of 50 pediatric patients involved in this study, $5(10 \%)$ patients had a history of birth asphyxia.

Distribution of subjects according to the mode of delivery: Out of 50 pediatric patients involved in our study, 19 (38\%) were born by LSCS (Lower segment cesarean section) and $31(62 \%)$ were born by vaginal delivery.

Distribution of cases on the basis of presence or absence of abnormal findings on MRI brain: MRI brain revealed abnormalities in 18 (36\%) cases while MRI study of remaining $32(64 \%)$ was normal.
Distribution of MRI abnormalities according to age: The most common findings in the age group 16 years were inflammatory granuloma. Mesial temporal sclerosis and focal cortical dysplasia were observed in the age group of more than 12 years only. Tuberous sclerosis, hemorrhage, lissencephaly, arachnoid cyst, and cerebral atrophy were observed in the age group of 0-1 year.

Distribution of patients according to different MRI findings: The most common MRI brain finding was inflammatory granuloma which was observed in $5(10 \%)$ patients. The next most common finding was a hypoxic-ischemic injury which was present in $4(8 \%)$ of patients. Other findings were:- Mesial temporal sclerosis in 2(4\%), Arachnoid cyst in 1 $(2 \%)$, cerebral atrophy in $1(2 \%)$, Focal cortical dysplasia in 1 (2\%), Joubert syndrome in $1(2 \%)$, Lissencephaly in $1(2 \%)$, hemorrhage in $1(2 \%)$ and Tuberous sclerosis in $1(2 \%)$ case.

Multicystic Encephalomalacia-Spectrum of Hypoxic Ischemic Injury

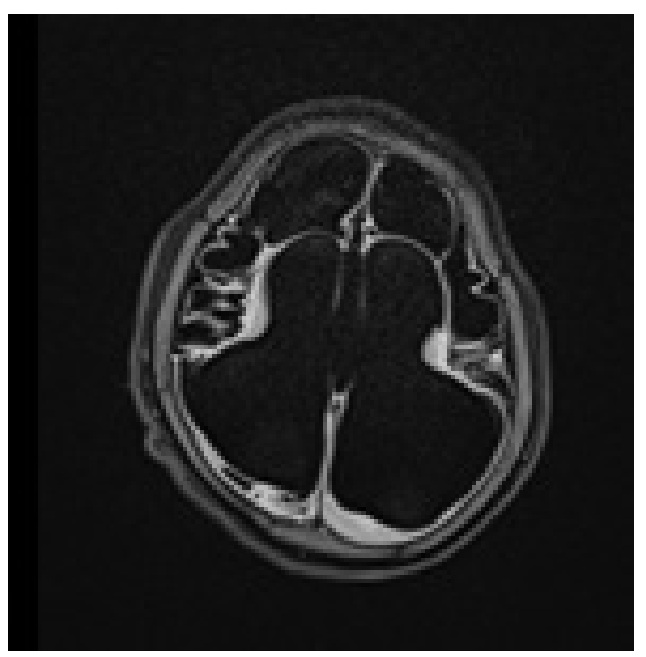

Fig-1 (A)

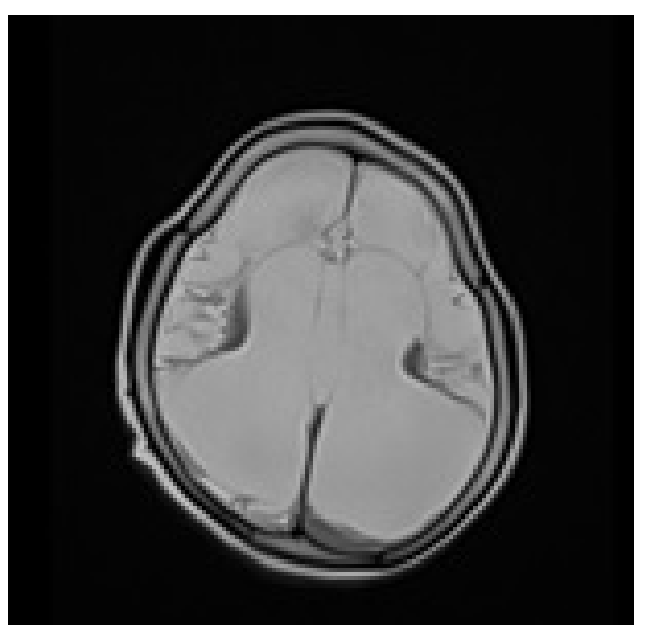

Fig-1 (B) 


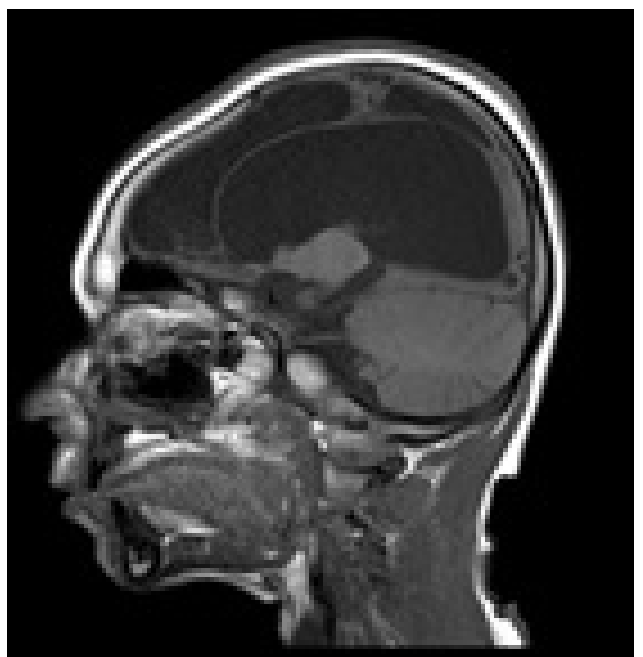

Fig-1 (C)

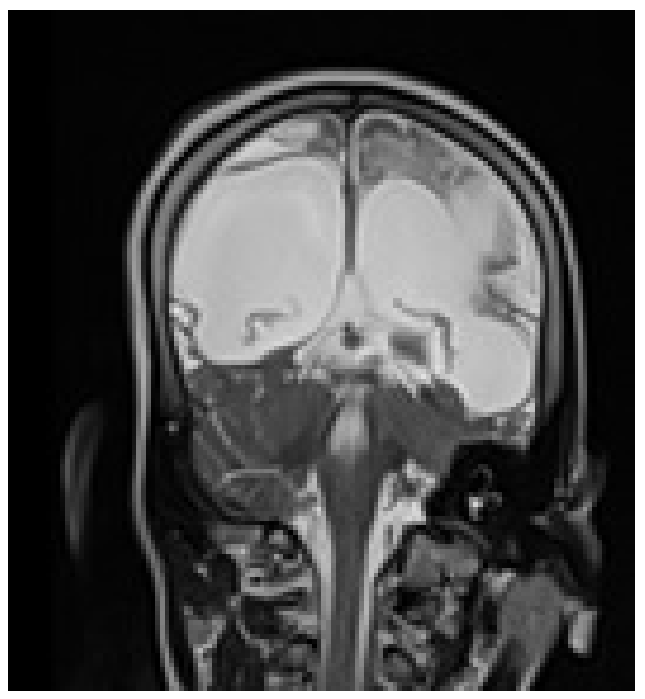

Fig-1 (D)

Fig-1: (A) Axial Flair T2 ;1(B) T2 axial; 1(C) T1 sagittal D) T2 coronal - large part of cerebrum is seen replaced by cystic spaces with exvacuo dilatation of bilateral lateral ventricles and third ventricle- spectrum of hypoxic ischemic injury.

\section{Mesial Temporal Sclerosis}

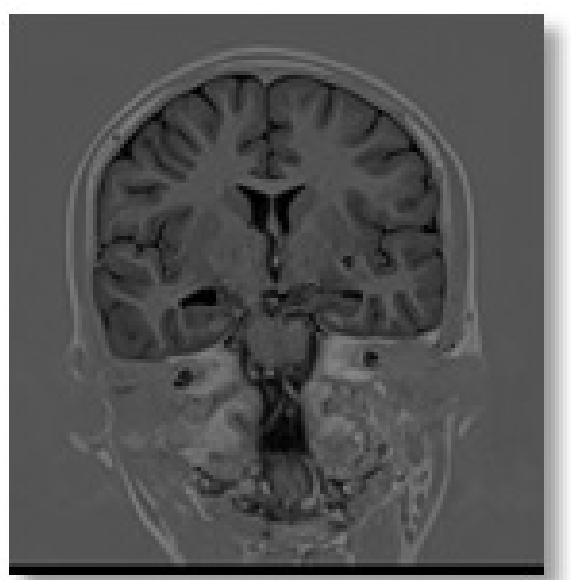

Fig-2 (A)

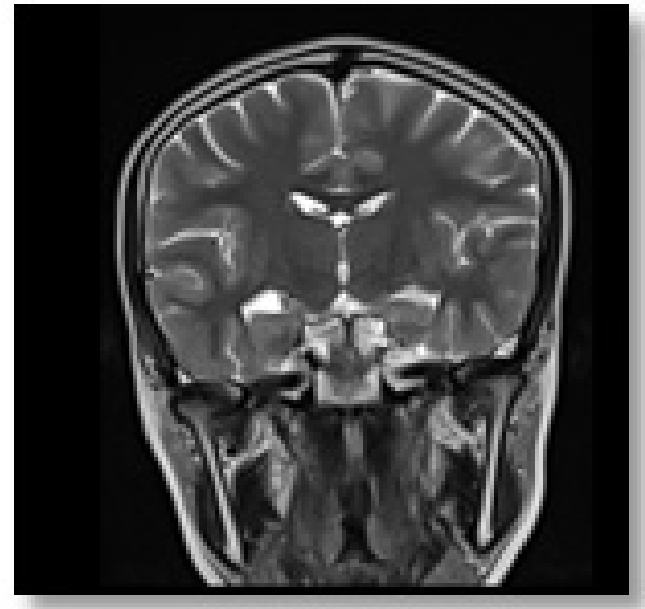

Fig-2 (B)

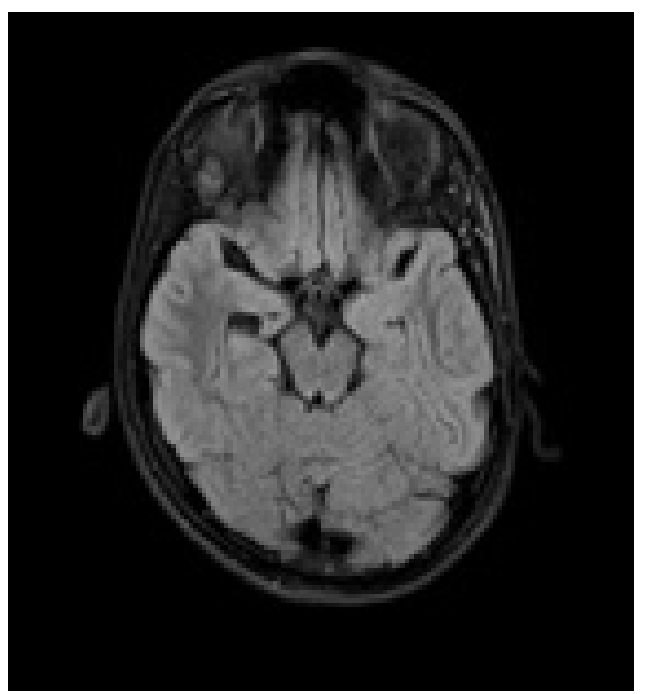

Fig-2 (C)

Fig-2: (A) Coronal MPRAGE, (B) Coronal T2 W image, (C) Axial FLAIR image- The right hippocampus appears reduced in volume. The temporal horn of the right lateral ventricle and the choroid fissure are prominent as compared to the left side. Findings are indicative of right mesial temporal lobe sclerosis.

Distribution of MRI findings according to sex: The most common findings on MRI in male patients was hypoxic-ischemic injury and in female patients, the most common abnormality was inflammatory granuloma. 1 case of cerebral atrophy was detected in a female patient and no case of focal cortical dysplasia, arachnoid cyst, hemorrhage, or hypoxicischemic injury was found among the female patients involved in the study. 1 case of focal cortical dysplasia was detected in a male patient and no case of cerebral atrophy, Joubert syndrome, 
Lissencephaly or tuberous sclerosis was detected among the male patients involved in the study.

\section{Table-1: Distributions of patients according to} different MRI findings

\begin{tabular}{|l|l|l|}
\hline \multicolumn{1}{|c|}{ MRI Findings } & No. of cases & Percentage \\
\hline Arachnoid cyst & 1 & 2.0 \\
\hline cerebral atrophy & 1 & 2.0 \\
\hline Focal Cortical Dysplasia & 1 & 2.0 \\
\hline Joubert Syndrome & 1 & 2.0 \\
\hline Lissencephaly & 1 & 2.0 \\
\hline Hemorrhage & 1 & 2.0 \\
\hline Tuberous Sclerosis & 1 & 2.0 \\
\hline Mesial Temporal Sclerosis & 2 & 4.0 \\
\hline Hypoxic Ischemic Injury & 4 & 8.0 \\
\hline Inflammatory granuloma & 5 & 10.0 \\
\hline Normal study of Brain & 32 & 64.0 \\
\hline Total & 50 & 100.0 \\
\hline
\end{tabular}

Distribution of MRI findings according to gestational age at birth: In the present study, one case of hypoxic-ischemic injury was seen in preterm children and three cases of hypoxicischemic injury were seen in patients born at term. Two cases of mesial temporal sclerosis were seen in patients born at term while no case was seen among patients born at preterm. 1 case of focal cortical dysplasia was detected in a preterm born patient while no case of Joubert syndrome, hemorrhage or tuberous sclerosis was detected amongst preterm born patients. One case each of Joubert syndrome and hemorrhage was detected in patients born at term while no case of an arachnoid cyst, cerebral atrophy, focal cortical dysplasia or lissencephaly was detected amongst term-born patients.

Distribution of MRI findings according to the history of birth asphyxia: In the present study, there was a total of 4 cases of hypoxic-ischemic injury, out of which 3 patients had a history of birth asphyxia. Amongst the 5 cases of inflammatory granuloma, none of the patients had a history of birth asphyxia. There was 1 case of an arachnoid cyst, 1 case of cerebral atrophy, 1 case of Joubert syndrome, 1 case of hemorrhage and 1 case of tuberous sclerosis, all of which had no history of birth asphyxia.

Distribution of MRI abnormalities according to the mode of delivery: There was a total of 4 cases of hypoxic-ischemic injury out of which 2 patients were delivered by LSCS (low segment cesarean section) and the other 2 were delivered by NVD (normal vaqinal delivery). There was 1 case of
Lissencephaly which was delivered by NVD. Amongst the 5 cases of inflammatory granuloma 4 cases were delivered by NVD. 1 patient had an arachnoid cyst, 1 patient had cerebral atrophy, 1 patient had focal cortical dysplasia, 1 patient had Joubert syndrome and 1 patient had tuberous sclerosis, and all of these patients were delivered by LSCS.

MRI in cases of Inflammatory granulomas: Amongst the 5 cases of inflammatory granulomas, 3 (60\%) patients had tuberculoma, 2 (40\%) patients had neurocysticercosis.

Statistical significance of the history of birth asphyxia for abnormalities in MRI findings: There was a total of 5 cases with a history of birth asphyxia and all of them had an abnormality in the brain which was statistically significant as described in the below-mentioned table ( $p$-value <0.005).

Table-2: Statistical significance of the history of birth asphyxia for abnormalities in MRI findings

\begin{tabular}{|l|l|l|l|l|c|}
\hline \multirow{2}{*}{$\begin{array}{c}\text { History of Birth } \\
\text { Asphyxia }\end{array}$} & \multicolumn{2}{c|}{ MRI } & Total & $\begin{array}{c}\text { Chi-square } \\
\text { value }\end{array}$ & $\begin{array}{c}\text { p- } \\
\text { value }\end{array}$ \\
\cline { 2 - 5 } & Abnormal & Normal & & & \\
\hline No & 13 & 32 & 45 & & \multirow{2}{*}{0.004} \\
\hline Yes & 5 & 0 & 5 & 9.877 & \\
\hline Total & 18 & 32 & 50 & & \\
\hline
\end{tabular}

\section{Discussion}

Nearly $4-6 \%$ of children are expected to have a seizure episode by age of 16 years. Amongst these, about $70 \%$ of these patients are admitted and have to undergo several investigations [3]. MRI is an important non-invasive imaging modality in the management of seizures which reveal pathologies of the brain conveniently. Although a good number of studies have been conducted on adults in India, only a few studies regarding the spectrum of MRI abnormalities in pediatric patients with seizures are available and therefore it was important to study various abnormalities on MRI brain in pediatric patients with seizures. In the present study abnormal neuroimaging was found in 18 (36\%) cases while in the rest of the cases no abnormality was detected in the brain. Our findings are similar to that of a study conducted by Kramer et al (1988) which detected neuroimaging abnormalities in 35\% of patients [24]. Our study results are also consistent with a study done by Sahdev et al (2016) which revealed abnormal neuroimaging in $31.4 \%$ cases while in $68.6 \%$ of cases no abnormality was seen [1]. However in a study conducted by Gulati 
Et al (1991), the study revealed abnormal neuroimaging in $92.9 \%$ of the cases [13]. These findings did not correspond to our study. In our study, the most common cause of seizures as identified by MRI brain was inflammatory granuloma (in $10 \%$ cases) while the second most common was a hypoxic-ischemic injury in $8 \%$ of patients. A similar study by Sahdev et al was conducted in India and it showed the most common cause of seizure in the age group of 0 months to 12 years was Inflammatory granuloma which included both tuberculoma and neurocysticercosis [1]. In our study, the percentage of CNS tuberculosis is considerable and is $6 \%$, followed by Neurocysticercosis which was in $4 \%$ of cases. The studies conducted by other developing countries such as Nigeria, Africa, etc. also demonstrated similar findings [25]. Such findings could be attributed to the low socio-economic status of people and poor hygiene and lack of proper sanitation facilities, still at large in developing countries. But in a study conducted by Kalnin et al (2008), demonstrated the most common cause of seizures was ventriculomegaly $(51 \%)$, followed by leukomalacia/ gliosis (23\%) as the second most common cause [26]. In our study, there was one $(2 \%)$ case of tuberous sclerosis. On MRI scan, subependymal nodules and cortical tubers were detected. These results are similar to that of the study conducted by chaurasia et al which too revealed tuberous sclerosis in only one $(0.5 \%)$ case. Thus, it indicates that tuberous sclerosis is a rare cause of seizures [25]. Seizures are relatively uncommon presentation in cases of a brain tumor [27]. In our study, no case of any brain tumor was found. These results are similar to that of a study conducted by Shadev et al which also did not detect any brain tumor [1]. However, these results are different from that of the study conducted by Chaurasia et al (2013) which revealed a brain tumor in $4(2.1 \%)$ cases [25]. The most common cause of seizures in children in our study was inflammatory granuloma which included both tuberculoma and neurocysticercosis, both of which cannot be diagnosed without neuroimaging and are a potentially treatable cause of seizures. Therefore, timely imaging in such patients is of utmost importance. It allows the earlier initiation of treatment and also prevents recurrence of seizures. Detection of such causes well in time is of utmost importance and timely and accurate treatment based on MRI findings allows the treating physician to prevent the condition from worsening.
Neuroimaging is also helpful in such cases in deciding when to stop the treatment.

\section{Conclusions}

The MRI was able to identify brain lesions in $36 \%$ of pediatric patients who presented with seizures.

\section{What the study add to the existing knowledge}

The study revealed inflammatory granuloma as the commonest cause of seizures in children, followed by Hypoxic-Ischemic Injury. Early recognition of potentially treatable diseases helps in timely treatment and arrest of disease progression. It is recommended to use MRI as a primary investigation during the evaluation and management of pediatric seizures. MRI has also the advantage of being a radiation-free imaging modality however its high cost and availability are few of the limitations.

\section{Author's contribution}

Dr. Manoj Mathur Dr. Simran Jeet Singh, Dr. Navpreet Kaur, Dr. Dimple Mittal, Dr. Yogesh Garg, Dr. Ankit Raikhy contributed equally in the conduct of the study and in the preparation of the manuscript.

\section{Reference}

01. Sahdev R, Rao A, Sinha S. Neuroimaging in pediatric seizures. Int $\mathrm{J}$ Res Med Sci. 2017;5(1)295-299.

doi: [Article] [Crossref]

02. Johnston MV. Seizures in childhood, InKliegman RM, Behrman RE (eds) Nelson textbook of paediatrics. (18th ed) PhiladelphiaSaunders. 2010;2457-2470.

[Crossref]

03. Saini N, Baghel A. Neuroimaging abnormalities in children with first afebrile seizure. J Dent Med Sci. 2013;5(5)21-24.

[Crossref]

04. Silverstein FS, Jensen FE. Neonatal seizures. Ann Neurol. 2007;62(2)112-120. doi: [Article] [Crossref]

05. Clancy RR. Summary proceedings from neurology group on neonatal seizures. Pediatr. 2006;117(3)23-27. doi: [Article] [Crossref] 
06. Talebian A, Jahangiri M, Masoudi AN, Akbari H, Sadat $Z$. The etiology and clinical evaluations of neonatal seizures in Kashan, Iran. Iran J Child Neurol, Spring. 2015;9(2)29-35.

[Crossref]

07. Volpe J]. Neonatal seizures, In- Neurology of the Newborn. Philadelphia, PA- WB Saunders. $2001 ; 178-214$.

[Crossref]

08. Hallberg B, Blennow $M$. Investigations for neonatal seizures. Semin Fetal Neonatal Med. 2013;18(4)196-201.

doi: [Article] [Crossref]

09. Ronen GM, Buckley D, Penney S, Streiner DL. Long-term prognosis in children with neonatal seizures- a population-based study. Neurol. 2007;69(19)1816-1822.

doi: [Article] [Crossref]

10. Tekgul H, Gauvreau K, Soul J, Murphy L, Robertson $\mathrm{R}$, Stewart $\mathrm{R}$, et al. The Current Etiologic Profile and Neurodevelopmental outcome of seizures in term new-born infants. Pediatr. 2006;117(4)1270-1280.

doi: [Article] [Crossref]

11. Leth $H$, Toft PB, Herning M, Peitersen B, Lou HC. Neonatal seizures associated with cerebral lesions shown by magnetic resonanace imaging. Arch Dis Child Fetal Neonatal Ed. 1997;77(2)F105-F110.

[Crossref]

12. Pal DK. Epilepsy control in the 21st centuryleave no child behind. Epilepsia. 2003;44(3)273-275.

doi: [Article] [Crossref]

13. Gulati P, Jena A, Tripathi RP, Gupta AK. Magnetic resonance imaging in childhood epilepsy. Indian Pediatri. 1991;28(7)761-765.

[Crossref]

14. McCoy B, Benbadis SR. Approach to refractory childhood seizures. Therapy. 2010;7(5)497-506. [Crossref]

15. Kohelet D, Shochat R, Lusky A, Reichman B. Risk factors for neonatal seizures in very low birth weight infants- population-based survey. J Child Neurol. 2004;19(2)123-128. doi: [Article] [Crossref]

16. Volpe JJ. Neonatal seizures, In- Volpe JJ (eds), Neurology of the newborn. (5th ed) Philadelphia- Saunders/Elsevier. 2008;203-245. [Crossref]
17. Glass HC, Sullivan JE. Neonatal seizures. Curr Treat Options Neurol. 2009;11(6)405-413. doi: [Article] [Crossref]

18. Silverstein FS, Jensen FE, Inder T, HellstromWestas L, Hirtz D, Ferriero DM. Improving the treatment of neonatal seizures- National Institute of Neurological Disorders and Stroke workshop report. J Pediatr. 2008;153(1)12-15. doi: [Article] [Crossref]

19. Jensen FE. Neonatal Seizures- An Update on Mechanisms and Management. Clin Perinatol. 2009;36(4)881-900.

doi: [Article] [Crossref]

20. Schmitt B, Wohlrab G, Sander T, Steinlein OK, Hajnal BL. Neonatal seizures with tonic clonic sequences and poor developmental outcome. Epilepsy Res. 2005;65(3)161-168. doi: [Article] [Crossref]

21. Idro R, Gwer S, Kahindi M, Gatakaa H, Kazungu $T$, Ndiritu $M$ et al. The incidence, aetiology and outcome of acute seizures in children admitted to a rural Kenyan district hospital. BMC Pediatr. 2008;8;5-15.

doi: [Article] [Crossref]

22. Rauch DA, Carr E, Harrington J. Inpatient Brain MRI for New-Onset Seizures- Utility and Cost Eff -ectiveness. Clin Pediatr. 2008;47(5)457-60.

doi: [Article] [Crossref]

23. Shaik SJM, Ratnavelu E, Balakrishnan $U$, Amboiram $\mathrm{P}$, Ninan B, Chandrasekharan A. Spectrum of magnetic resonance imaging abnormalities in neonatal seizures in a tertiary care hospital in india. Int J Contemp Pediatr. 2016;3(4)1150-1155.

doi: [Article] [Crossref]

24. Kramer U, Nevo Y, Reider-Groswasser I, Sheuer E, Meyer JJ, Leitner $Y$, et al. Neuroimaging of children with partial seizures. Seizure. 1998;7(2)115-118. doi: [Article] [Crossref]

25. Chaurasia $R$, Singh $S$, Mahur $S$, Sachan $P$. Imagingin pediatric epilepsy- spectrum of abnormalities detected on MRI. J Evol Med Dent Sci. 2013;19(2)3377-3385. doi: [Article] [Crossref]

26. Kalnin AJ, Fastenau PS, deGrauw $\mathrm{TJ}$, et al. Magnetic resonance imaging findings in children with a first recognized seizure. Pediatr Neurol. 2008;39(6)404-414.

doi: [Article] [Crossref] 
27. Ibrahim K, Appleton R. Seizures as the presenting symptom of brain tumours in children. Seizure. 2004;13(2)108-112.

doi: [Article] [Crossref] 